\title{
SONIC HELIUM DETECTORS IN THE FERMILAB TEVATRON
}

\author{
R. J. Bossert \\ Fermi National Accelerator Laboratory \\ Batavia, Illinois, 60510, USA
}

\begin{abstract}
In the Fermilab Tevatron cryogenic system there are many remotely located lowpressure plate relief valves that must vent large volumes of cold helium gas when magnet quenches occur. These valves can occasionally stick open or not reseat completely, resulting in a large helium loss. As such, the need exists for a detector to monitor the relief valve's discharge area for the presence of helium. Due to the quantity needed, cost is an important factor. A unit has been developed and built for this purpose that is quite inexpensive. Its operating principle is based on the speed of sound, where two closely matched tubes operate at their acoustic resonant frequency. When helium is introduced into one of these tubes, the resulting difference in acoustic time of flight is used to trigger an alarm. At present, there are 39 of these units installed and operating in the Tevatron. They have detected many minor and major helium leaks, and have also been found useful in detecting a rise in the helium background in the enclosed refrigerator buildings. This paper covers the construction, usage and operational experience gained with these units over the last several years.
\end{abstract}

KEYWORDS: Sonic, Resonant, Concentration, Detector

PACS: $51.40 .{ }^{+} \mathrm{p}$

\section{INTRODUCTION}

The speed at which sound moves through a medium is dependent upon several variables, any of which will influence the time of flight of a pressure wave from its source to its destination. Of the three mediums (gas, liquid, and solid) sound waves travel the slowest through gases, faster through liquids, and fastest through solids. For our purposes in discussing the sonic helium detector units, we are mainly concerned with sonic velocity in atmospheric air and how it is affected by temperature, humidity and the introduction of a small amount of helium. An increase in any of these three causes the velocity of sound 
waves to increase, decreasing the time of flight. In the detectors we eliminate the variables of temperature and humidity by utilizing a reference tube, which leaves only the change due to helium.

\section{OPERATING PRINCIPLE}

The detector operates by comparing the acoustic resonant frequency of two closely matched tubes. One tube is designated as the reference and the other as the sample. Each tube has a speaker at one end and a microphone at the other. Each tube also has two holes located at the mid point for the purpose of drawing a small amount of sample or reference gas through them. Both tubes are enclosed in the cell, which is airtight and has three ports on it. One is for connecting a suction pump and the other two are used for bringing in the reference and sample gases from the outside world. The reference tube has atmospheric air drawn through it usually from the area where the detector is located. The sample tube has gas drawn through it that is from the area to be sampled, such as the discharge area of a relief valve. The cell is internally plumbed such that one side of each resonant tube is common with the internal cell volume, which is also connected to the pump port. The other side of each resonant tube is directly connected to either the sample or reference port. The pump sucks on the common cell volume, which causes a small flow to be drawn through each resonant tube. The speakers are both driven by the same phase electrical signal, so as long as the gas in both resonant tubes is identical, the signal picked up by both microphones are exactly in phase. For best sensitivity a phase locked loop circuit keeps the speaker signal at the resonant frequency of the reference tube. When the density of the gas in one of the tubes changes, the time of flight of the sound waves change, which appears as a phase shift between the microphone signals. The magnitude of the phase shift is converted to an analog signal, which is then compared to an error set point to determine the state of the alarm relay output.

\section{DESIGN CONSIDERATIONS}

\section{Temperature}

The units must be able to start up and operate throughout the full range of anticipated outside temperatures (approx. $-30^{\circ} \mathrm{C}$ to $50^{\circ} \mathrm{C}$ ), which causes several concerns. The air pump bellows becomes too stiff to pump effectively when very cold. Secondly, if a unit is powered up at a very cold temperature, it will take an unsatisfactorily long time to reach stable operating parameters. Thirdly, despite the fact that the circuitry compensates for temperature variations, the characteristics of the speakers and microphones versus temperature are not consistent. It is also necessary that the units stay dry. For these reasons a means of temperature regulation is necessary. It consists of a 100 -watt heater with a variable duty cycle circuit to control it and a thermistor to sense temperature. It is set to keep the inside temperature at approximately $46^{\circ} \mathrm{C}$. In order to minimize potential errors due to the resonant tubes being at different temperatures, approximately one meter of copper tubing is used to connect the sample and reference ports where they enter the detector to where they connect to the cell input ports. The two tubes are soldered together to act as a heat exchanger. The integrated circuits used in the units are all military spec temperature range so that even after an extended power outage in the coldest ambient temperature, the heater circuit will function at startup. 


\section{Microphone Signal Filter}

The microphones have good low frequency sensitivity, and the pulsations produced by the pump overwhelm the phase comparison circuitry if fed directly to the comparators. Unfiltered, the microphone signals appear as an approximately $1200 \mathrm{~Hz}$ sine wave superimposed on a much larger $60 \mathrm{~Hz}$ sine wave. The $60 \mathrm{~Hz}$ component is produced by the pressure pulsations of the vibrating pump. An active filter attenuates it to a manageable level while not affecting the primary frequency at which the cell tubes resonate.

\section{High Levels of Helium}

These units are set to alarm when the concentration of helium in air exceeds $2 \%$. The alarm at that point is triggered by the degree of phase shift between the microphone signals. A problem was observed during testing, however, where the units would go into alarm state as the helium level increased, but as it kept increasing the unit would clear again. We found that as the helium concentration increased, the amplitude of the signal picked up by the sample microphone decreased. When the signal to the sample comparator got very low it would quit functioning. The output of the comparator would then go low. Since there was no longer a phase shift between the signals, the unit would then clear. This undesirable behavior was corrected by slightly biasing the positive leg of the sample comparator, making it go full on and forcing the circuitry into the alarm state in the absence of a sample microphone signal.

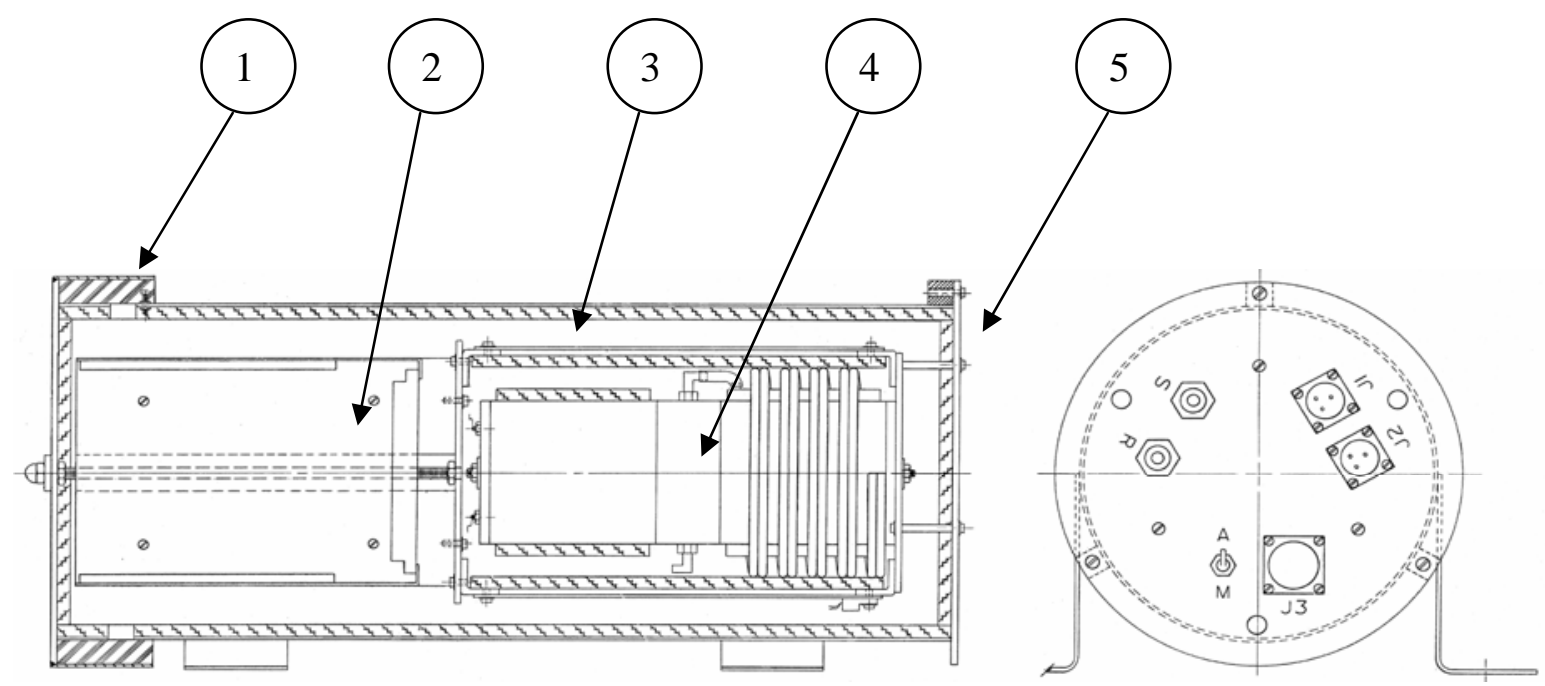

FIGURE 1. A cross-sectional view of a complete unit. \#1 top cover, \#2 pump board and main circuit board holder, \#3 heater, \#4 cell, \#5 bottom plate with plumbing and electrical connections. 


\section{PHYSICAL DESCRIPTION}

The units are housed in an aluminum tube that is $44.45 \mathrm{~cm}$ long and $17.78 \mathrm{~cm}$ in diameter. There is a plate on one end that has the electrical connectors and the reference and sample port connections. The units operate on 120VAC @ less than .5 amp and provide a discrete alarm signal that has an adjustable set point between approximately 0 and 4\%. Various other analog signals are available through another connector that can be read externally to determine if the unit is operating correctly. The resonant cell tubes are $15.42 \mathrm{~cm}$ long and $2.54 \mathrm{~cm}$ in diameter. The units vary somewhat with ambient conditions, but generally the cell frequency runs between 1150-1200 Hz. The sample and reference gas port connections are $6.35 \mathrm{~mm}$ O.D. They should be mounted with the electrical and plumbing connector end down with enough room at the top to be able to remove the cover for field service.

\section{MAIN COMPONENT DESCRIPTION}

\section{Air Pump}

The pumps ordinarily provide at least two or three years of service. They are mounted on a circuit board that can be easily removed from the unit. Their design is similar to that of an aquarium vibrator pump and even utilizes some of the components of a commonly available model.

\section{Microphones}

The sound pickups used are PC mount condenser microphone elements. They are a common microphone element probably found in a lot of lower cost hand held microphones. They are glued in place in the nylon tube end cap with high strength RTV.

\section{Speakers}

The speaker used is a relatively low quality earphone similar to what was commonly supplied with a transistor radio in years past. It is also glued in place with high strength RTV.

\section{Heater}

The heater is a standard 100-watt silicone rubber heating element wrapped around a $13.33 \mathrm{~cm}$ diameter aluminum tube. It surrounds the cell and the heat exchanger that equalizes the temperature of the incoming reference and sample gas.

\section{Cell}

The cell enclosure is airtight and contains the two resonant tubes, designated the sample and reference. During assembly the individual cell tubes are tested and matched as well as possible in terms of having the same resonant frequency. On one end the cell has electrical feed through connections for the speaker and microphone signals. It is the heart of the detector and requires the most care to assemble. 


\section{Main Circuit Board}

The main circuit board has the voltage controlled oscillator (VCO), the speaker driver, the active filter, phase locked loop circuitry, the microphone phase comparison and the heater control circuitry. It has potentiometers for parameter adjustment that are accessible with the unit operating if the top cover is removed. The circuit board can be easily removed.

\section{Pump Board}

The pump board has the air pump, the 5 Vdc power supply, a solid-state relay to switch power for the heater and a DIP style relay for external alarm annunciation. It is easily removable from the top if the cover is removed.

\section{CIRCUITRY OPERATION}

The VCO drives the base of a 2N2219 transistor to provide enough power to drive the speakers. The signal sent to the speakers is also sent a comparator and timer IC to produce charge pulses if needed to maintain the VCO output frequency at resonance. The charge pulses are rectified, integrated and fed to the control input of the voltage-controlled oscillator as an analog signal. The VCO automatically adjusts to stay at the resonant frequency of the reference tube. The reference and sample microphone signals are then high-pass filtered to remove the $60 \mathrm{~Hz}$ component and sent to comparator circuits to produce square waves. The reference and sample signals are sent to opposite polarity inputs on the comparators so that the output of the two comparators is out of phase. These two signals are then fed to a two input AND gate. If the two microphone signals are exactly in phase, as they are when no helium is present, the square waves coming from the microphone comparators look like two nearly perfectly out of phase square waves, which produces no output from the AND gate. As the signals move increasingly out of phase, the AND gate produces pulses of increasing width. These pulses are then rectified and integrated to become an analog signal that increases with increasing phase shift. This analog signal is then fed to the positive input of another comparator. The voltage at the negative leg of this comparator is adjustable via a potentiometer, and its setting determines what degree of phase shift causes the unit to go into alarm state.

\section{INSTALLATION DETAILS}

The units are intended to be installed on a wall with the connector end down. They were designed and tested to operate properly thoughout the temperature range of $-30^{\circ} \mathrm{C}$ to $50^{\circ} \mathrm{C}$. If the unit is outside, it is very cold and if power is lost for several hours, they may take two hours to stabilize and warm up enough for the pump to work properly. For the sample tubing clear $6.35 \mathrm{~mm}$ I. D. tubing is most commonly used. The sample tube intake must be positioned such that water cannot get sucked into it. A water drop in the inlet tube can result in false alarm indications. If water is sucked into the cell in sufficient quantity, it will destroy the mics and/or speakers. The length of the inlet tubing should be as short as practical for fastest response time, and can be vertical or horizontal. Intake tubing as long as eight meters has been used with response times staying under two minutes. 
If the unit is mounted in an area where the relative humidity is anticipated to be very different from the relative humidity of the sample gas, consider running a reference tube to the same general area as the sample tube in order to avoid errors due to a difference in density because of a difference in relative humidity.

Finally, do not route the sample tubing such that it contacts a cold surface. The moisture in the air will condense in the cold tubing and create problems.

\section{OPERATING EXPERIENCE}

The first five detectors were installed in the field in 1989. They were installed at the central helium liquefier as a test case to monitor the suction relief valve port of the main Worthington compressors and the relief valve port of the gas recovery system. They worked well there, alarming and clearing reliably when system perturbations would occasionally cause a relief valve to open. After this, 24 more were installed in the satellite refrigerator buildings, one in the anti proton source refrigerator and nine in the ring compressor buildings for a total of 39. We plan to install at least five more.

The Fermilab Tevatron is a little over four miles in circumference with the cryogenic system being divided into 24 sections, or "houses". When a magnet quench occurs, we typically see anywhere from 0 to nearly every single ring unit go briefly into alarm state and clear within a few minutes. The most usual case is a single house quench that may cause two or three houses upstream and downstream to briefly alarm. When quenches occur, the velocity of the gas can blow nuts, bolts or anything else out of the relief port which can either damage the sealing surface or get stuck in the relief valve exhaust flange. We have seen several times where a plate relief continues to "weep" after it has opened, causing an intermittent or steady alarm that does not clear until the sealing surface is cleaned. When the units were originally installed on the refrigerator house plate reliefs it was at first thought that many of the units were malfunctioning because the analog error signal appeared noisy, but an inspection revealed that most of the noisy locations had dirt on the o-ring and/or the sealing surface. All units have three signals connected to the Tevatron control system for remote readout, annunciation and logging purposes. They are the discrete alarm output, the analog error signal, and the VCO analog signal. In the early days we thought that it would only be necessary to read back the discrete alarm, but have found that the additional two analog signals give you much more insight as to whether the unit is operating properly, and also the character of a leak. We have also found an unexpected benefit where we can detect a problem inside the enclosed refrigerator buildings by seeing a rise in the VCO signal. We have seen this on several occasions when an expansion engine fails. On one occasion when a quench occurred and a component in the Tevatron tunnel ruptured, we were able to see the helium wave move. As it entered the area of a refrigerator building it would rise up through the tunnel penetration and collect in the frig house directly above, causing the VCO signal to rise.

As far as reliability, the detector units are generally very good. The pump is by far the most frequent problem, the most common failure mode being that a crack develops in its rubber bellows after two or three years. When the pump is failing, the units respond slowly or not at all and may take a long time to clear. Fortunately the pump board is easily replaceable in the field and requires no adjustment to the unit after the change out.

In the early days we also saw a few instances where the cell would fail. It would go to alarm state and you would no longer get usable signals from one or both microphones. An autopsy of the failed cells revealed that they had ingested water in sufficient quantity to destroy the microphone element, and usually caused the steel earphone diaphragm to rust 
extensively. We found that this was due to either the units sucking water in directly from rain, or the sample tube being in contact with a cold pipe. When the sample tubing is chilled below the freezing point of water and the air being drawn through it is humid, you will plug the sample tube with ice in fairly short order. That in itself is obviously a problem, but it carries with it the further hazard that, when it warms up, the ice plug turns into water that is subsequently sucked into the cell.

\section{SUGGESTIONS FOR IMPROVEMENT}

\section{Circuitry}

The circuitry is fairly simple and can be improved in several areas. Some of the integrated circuits may not even be available any longer. The phase locked loop and the heater control circuit could both be simplified. If a different type of pump that does not produce pressure pulsations was found, the microphone filter circuitry could be eliminated.

\section{Pump}

The pump is the most troublesome part of these units. A type that does not produce pressure pulsations and has longer life would be beneficial.

\section{Resonant Tubes}

Make one or both resonant tubes adjustable in length such that you can tune them. This would eliminate the cumbersome matching process that is necessary as part of the cell assembly.

\section{Speakers and Microphones}

Use speakers and microphones that are not damaged by water, possibly a miniature voice coil dynamic type that has a plastic cone. It may be possible to use the same unit for the speaker and, at the other end of the tube, as a sound pickup.

\section{SUMMARY}

A unit for detecting a helium concentration on the order of two percent in air has been developed and is in use around the Fermilab Tevatron. Its operating principle is based on the difference in sonic velocity between two resonant tubes when helium is introduced into one of them. The units provide an adjustable discrete alarm point and have analog signals externally available for either monitoring operating conditions or for repair diagnostics. They are built using commonly available and very inexpensive components. They have saved money many times by detecting stuck or leaking plate relief valves and thereby minimizing losses of helium. They ordinarily require maintenance no more often than two years or so, with the most usual being a pump replacement which can easily be done with the unit in place in the field. They require a significant amount of time and expertise to assemble. 


\section{ACKNOWLEDGEMENTS}

Fermilab is operated by Universities Research Association Inc. under contract No. DE-AC02-76CH03000 with the U. S. Department of Energy.

I wish to thank Dr. Ronald J. Walker for the original concept and early design work. I also thank William Martin, Joe Brown, John Juneau, Carl Staples, Mike Dinnon, Rich

Ferry, Darryl Richardson and Dave Richardson for early prototype, assembly and documentation assistance. 